\title{
Normally Transparent Tribo-Induced Smart Window
}

Jiaqi Wang ${ }^{\dagger, \ddagger}$, Cuiling Meng§, Qian Gu ${ }^{\dagger, \#, ~ M a n ~ C h u n ~ T s e n g §, ~ S h u ~ T u e n ~ T a n g ~}{ }^{\S}$, Hoi Sing Kwok Jia Cheng ${ }^{\#}$, Yunlong $\mathrm{Zi}^{*+, \ddagger}$

†. Department of Mechanical and Automation Engineering, The Chinese University of Hong Kong, Shatin, N.T., Hong Kong, China

‡. Shun Hing Institute of Advanced Engineering, The Chinese University of Hong Kong, Shatin, N.T., Hong Kong, China

§. State Key Laboratory on Advanced Displays and Optoelectronics Technologies, Hong Kong University of Science and Technology, Clear Water Bay, Kowloon, Hong Kong, China.

\#. State Key Laboratory of Tribology, Department of Mechanical Engineering, Tsinghua University, Beijing 100084, China.

Email: ylzi@cuhk.edu.hk

1. Temperature profile of the alignment layer treatment: The substrate with coated film was firstly soft-baked at $80^{\circ} \mathrm{C}$ for $30 \mathrm{~s}$ and then cooled down at room temperature for $60 \mathrm{~s}$. During the cooling process, the surface temperature of the film was recorded every 1 second using a thermocouple thermometer.

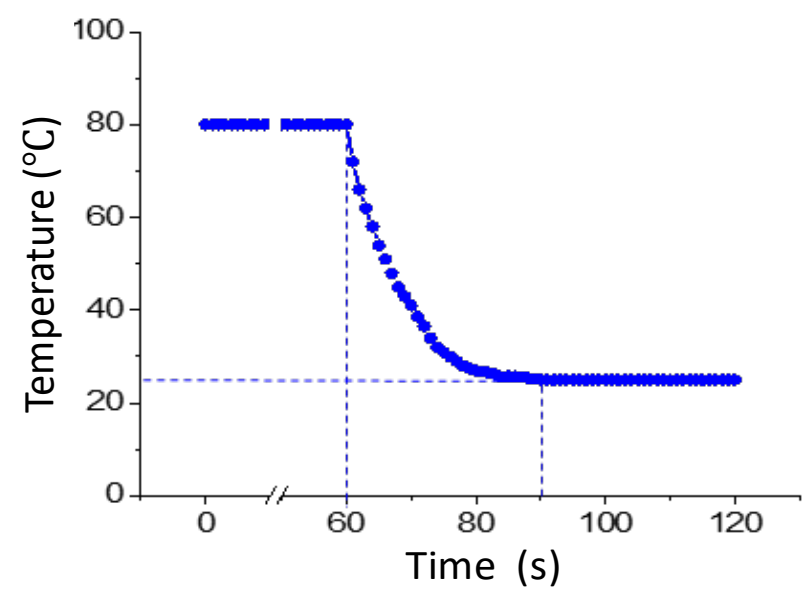

Figure S1. Temperature variation as a function of cooling time. 
2. Comparison between inhomogeneous and smooth surfaces: when mixture solution containing polyimide and LC monomer is coated on the substrate, the coated film after cooling process shows an inhomogeneous morphology with many puddle-like domains embedded. The domain size is around $10 \mu \mathrm{m}$ and the average domain depth is around $100 \mathrm{~nm}$. However, when pure polyimide solution is deposited, the film surface is so smooth that the surface roughness is within $1.0 \mathrm{~nm}$.

(a)

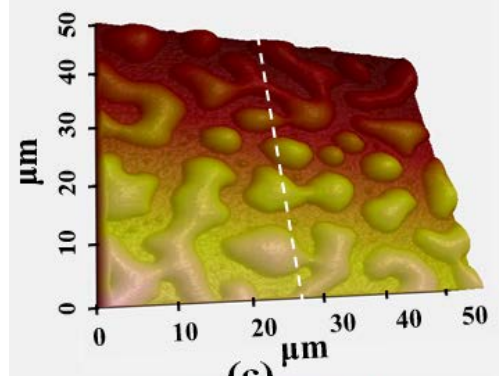

(c)

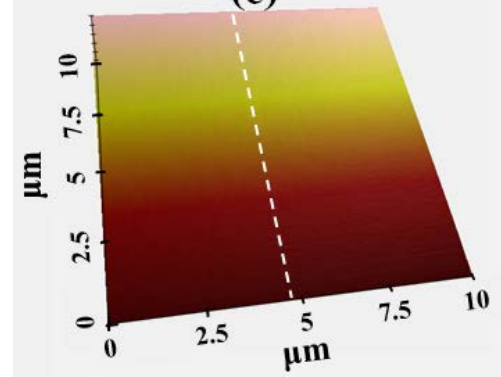

(b)

Line Profile

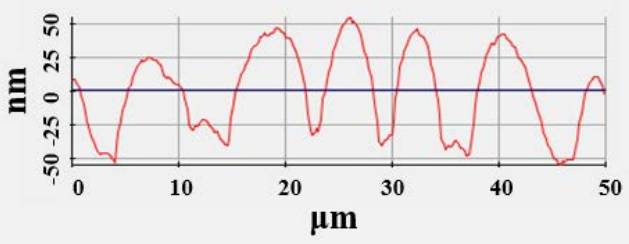

(d)

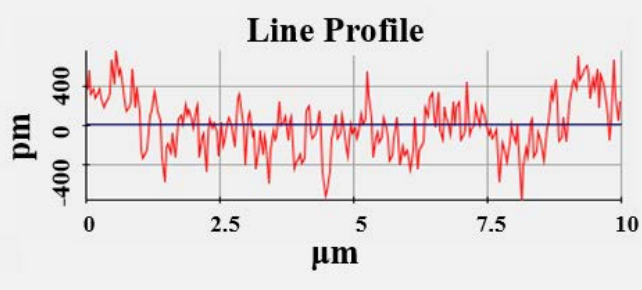

Figure S2. Alignment surface morphologies with (a) and without (c) inhomogeneity treatment, where the average depth is around $110 \mathrm{~nm}$ (b) and $1 \mathrm{~nm}(\mathrm{~d})$ respectively.

3. Mechanism of light scattering: The fundamental reason for light scattering is due to the mismatch of two or more than two materials. LC is a birefringence material and has two different refractive indices along its short-axis and long-axis. The refractive indices along short-axis and long-axis are called ordinary refractive index, $n_{o}$ and extra-ordinary index, $n_{e}$, respectively. Therefore, we can analyze the scattering in the power-off and -on states based on the refractive index of nametic LC and LC polymer when the light impinges into the PNLC window. As shown in Fig. S3 (a), in the off state, the incident light sees both ordinary refractive indices of nematic LC and LC monomer, abbreviated as $n_{o l}$ and $n_{o p}$, respectively. If the nametic LC is selected with its $n_{o l}$ close to $n_{o p}$, the light will not scatter. In the power-on state, the LC polymer keeps unchanged while the nametic LCs realign as a response to the electrical field. In this case, the incident light meets an updated $n_{\text {efl }}$ of the nematic LC and the unchanged $n_{\text {op }}$ of polymer (see Fig. S3 (b)). More specifically, the $x$-component of incident light sees $n_{1 e f l}$, which is expressed as: $n_{1 e f l}=$ $\frac{n_{o l} n_{e l}}{\sqrt{n_{e l} \sin ^{2} \theta_{1}+n_{o l} \cos ^{2} \theta_{1}}}$, and the $y$-component of incident light sees $n_{2 e f l}$, which is expressed as: $n_{2 e f l}$ $=\frac{n_{o l} n_{e l}}{\sqrt{n_{e l^{2}} \sin ^{2} \theta_{2}+n_{o l^{2}} \cos ^{2} \theta_{2}}}$. If $n_{1 e f l}$ and $n_{2 e f l}$ is not equal to or largely differs from $n_{\mathrm{op}}$, then the incident light will be strongly scattered. In addition, different LC molecules have different orientations (i.e., $\theta_{1}$ and $\theta_{2}$ as illustrated in Fig. S3 (b)), therefore, multiple scattering is expected. 
(a) Top view of off state

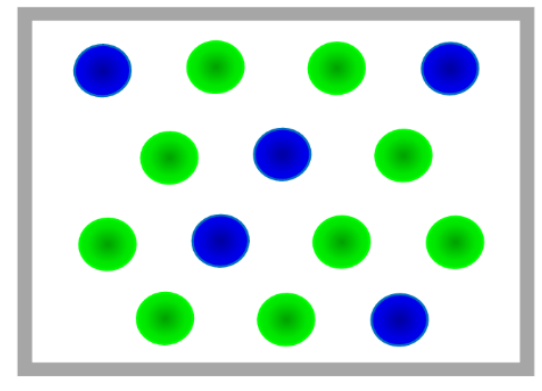

Nematic LC: $n_{o l}$ LC polymer: $n_{o p}$ $n_{o l} \approx n_{o p}$ (b) Top view of on state

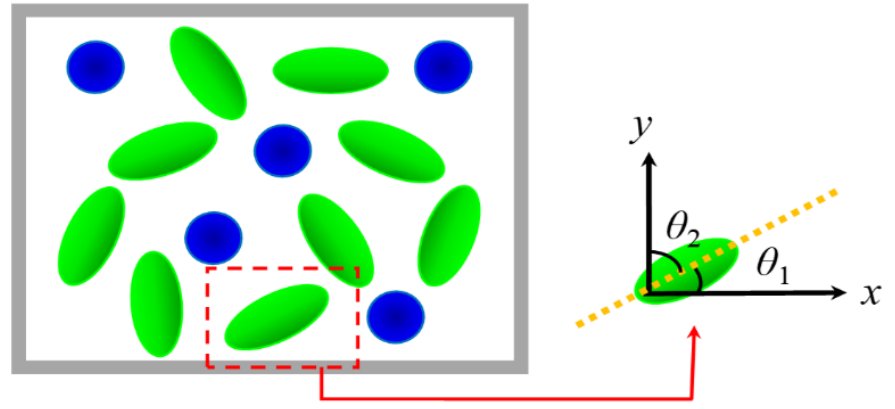

Nematic LC: $n_{\text {efl }}$

LC polymer: $n_{o p}$

$n_{\text {efl }} \neq n_{\text {op }}$

Figure S3. Distributions of nematic LC and LC polymer from the top view at power-off (a) and on (b) states.

4. Electrical characterization of the tribo-induced PNLC: A measurement setup was constructed to evaluate the electrical performance of the PNLC under the driving of the RFSTENG, where two Keithley 6514 electrometers, serving as a voltmeter and charge-meter, were connected to the PNLC in series and parallel, respectively, measuring the voltage drop of the PNLC and the amount of transferred charge.

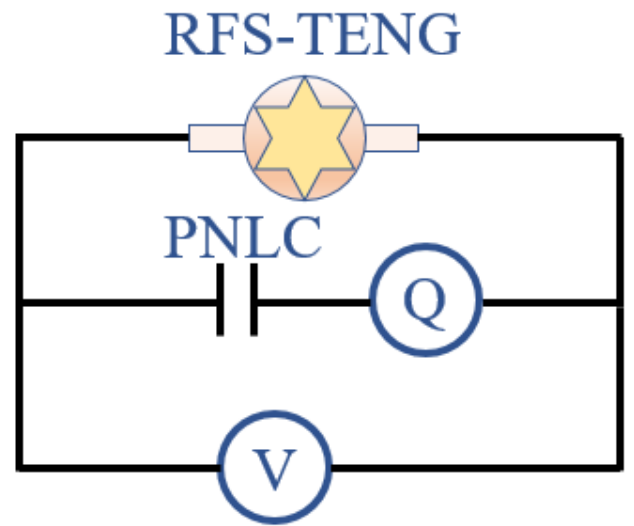

Figure S4. Measurement circuit to evaluate the electrical performance of the tribo-induced PNLC. 
5. Haze measurement: The definition of transmittance haze ratio is the ratio of the diffused transmittance to the total transmittance. Here, our haze ratio measurement system is established based on the earliest documentary standard ASTM D 1003, in which an integrating sphere are employed for the measurements as presented in Fig. S5 (a). By measuring the incident light with/without specimen and the scattered light by the specimen alone or by both the instrument and the specimen, one can calculate the haze ratio according to Eq. (2.1) to (2.3):

$$
\begin{gathered}
T_{\mathrm{t}}=T_{2} / T_{1} \\
T_{\mathrm{d}}=\left[T_{4}-T_{3}\left(T_{2} / T_{1}\right)\right] / T_{1} \\
\text { Haze }=T_{\mathrm{d}} / T_{\mathrm{t}} \times 100 \%
\end{gathered}
$$

where the meanings of $T_{1}, T_{2}, T_{3}$ and $T_{4}$ are listed in Fig. S5 (b).

(a)

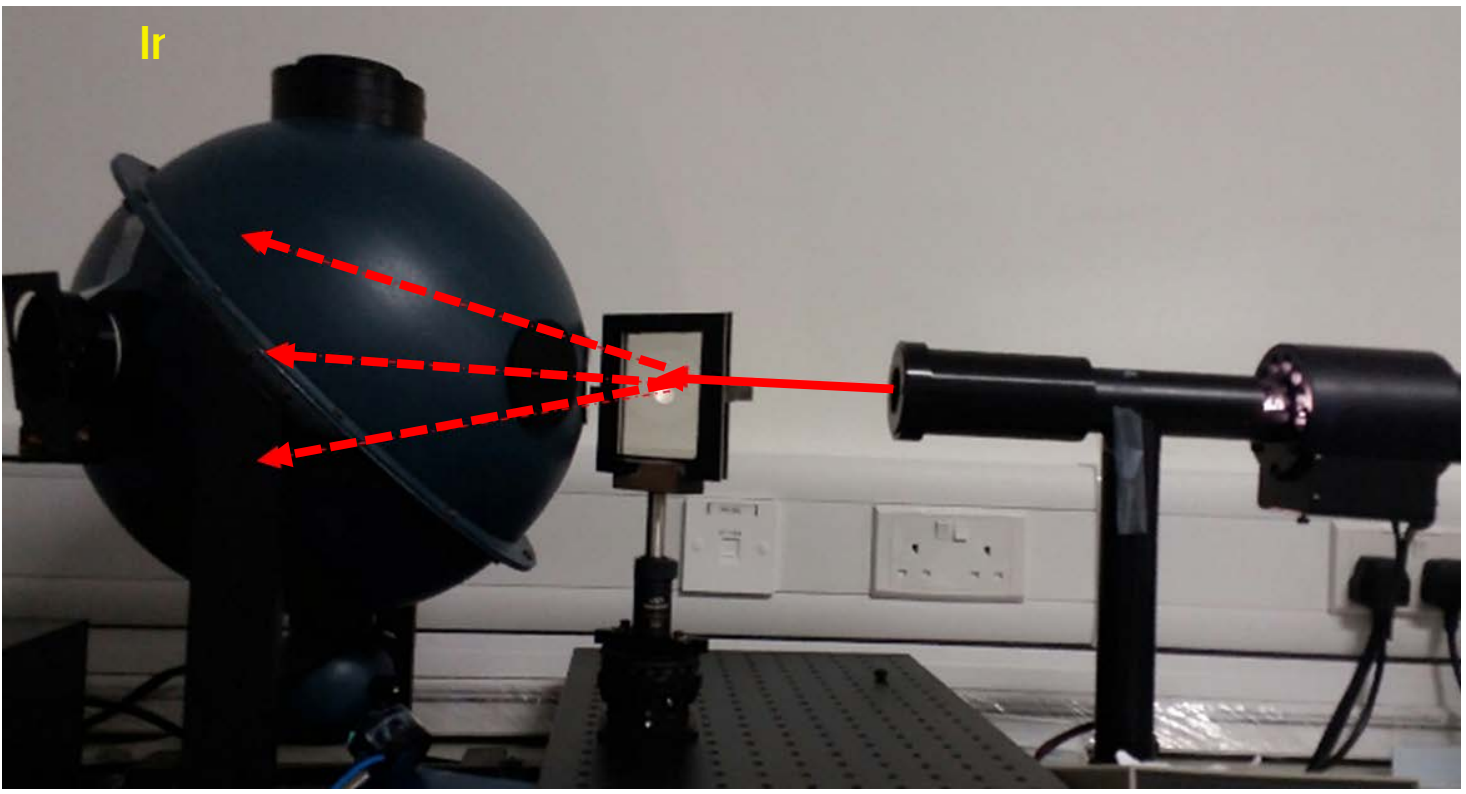

\begin{tabular}{l|l|l|l|l}
\hline (b) $\begin{array}{l}\text { Reading } \\
\text { designation }\end{array}$ & $\begin{array}{l}\text { Sample in } \\
\text { position }\end{array}$ & $\begin{array}{l}\text { Light trap } \\
\text { in position }\end{array}$ & $\begin{array}{l}\text { Reflectance } \\
\text { in position }\end{array}$ & Quantity represented \\
\hline $\mathrm{T}_{1}$ & no & no & yes & incident light \\
\hline $\mathrm{T}_{2}$ & yes & no & yes & $\begin{array}{l}\text { total light transmitted } \\
\text { by sample }\end{array}$ \\
\hline $\mathrm{T}_{3}$ & no & yes & no & $\begin{array}{l}\text { light scattered by } \\
\text { instrument }\end{array}$ \\
\hline $\mathrm{T}_{4}$ & yes & yes & no & $\begin{array}{l}\text { light scattered by } \\
\text { instrument and sample }\end{array}$ \\
\hline
\end{tabular}


Figure S5. (a) Setup of haze ratio measurement, which includes a collimated white light source, an integrated sphere and a photo-detector. (b) Parameters to be measured for haze calculation.

6. Response time measurement: In LC-type devices, the response time is mainly determined by the viscosity of LC material, the applied voltage as well as the cell gap. The setup of response time measurement is shown in Fig. S6 (a), in which the transmittance of PNLC window as well as the applied voltage loaded on PNLC window are simutaneously recorded using oscilloscope. Fig. S6 (b) shows the normalized transmission dynamic as a response to the driving signal of $60 \mathrm{~V} / 1 \mathrm{kHz}$ square voltage. The rising time from the clear state to the scattering state (an interval from $90 \%$ transmittance to $10 \%$ transmittance) only requires $0.5 \mathrm{~ms}$. However, the falling time, or relaxation time, was measured up to $6.5 \mathrm{~ms}$, much longer than the rising time. Nevertheless, the PNLC smart window is switched with no more than $7.0 \mathrm{~ms}$, which shows great potential on ultra-fast light valve and privacy film. Here, the fast response benefited from a weak compatibility between nametic LC and LC polymer. When LC molecules start to realign themselves as a response to the electric field, thousands of polymer strands will not exert much constraint on the rotating LCs so as to accelerate the LC reaction to electric field.

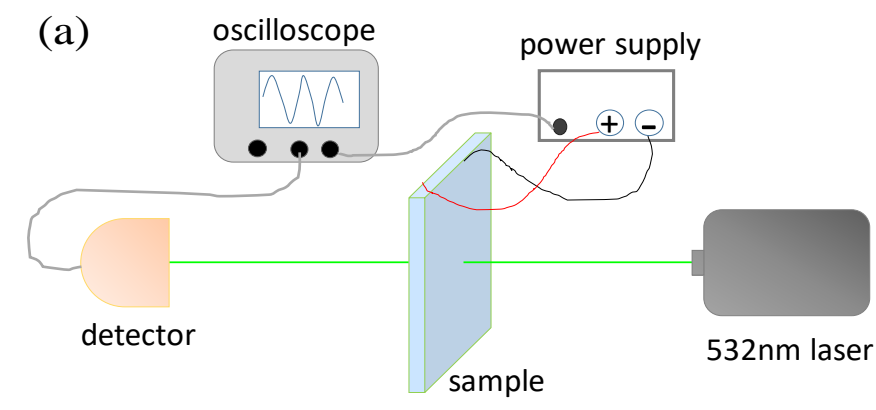

(b)

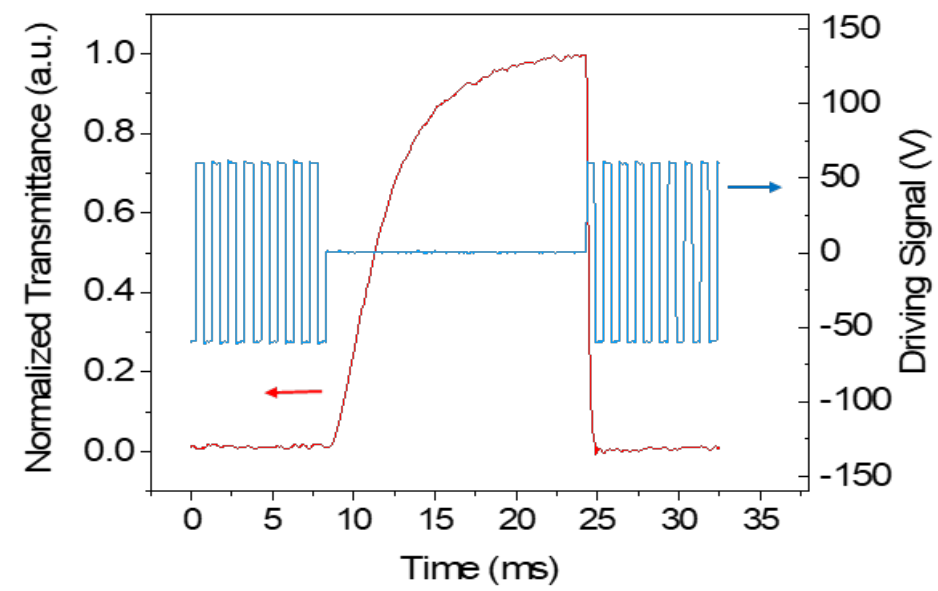

Figure S6. (a) Setup of response time measurement, which a green laser with $532 \mathrm{~nm}$ emitting wavelength, a detector, a PNLC sample, a power supplier as well as an oscilloscope; (b) The normalized transmittance dynamic (red curve) of PNLC smart window as a function of the driving signal (blue curve) with the amplitude of $60 \mathrm{~V}$ and the frequency of $1 \mathrm{kHz}$ square-wave voltage. 
Video. S1 Switching performance of the tribo-induced smart window (MP4)

Video. S2 Information protection application for QR coding scanning using the tribo-induced smart window (MP4)

Video. S3 Transparency variation of the tribo-induced smart window connected with different external capacitors (MP4) 